\title{
Use of motor learning principles to improve motor adaptation in adult obesity ${ }^{*}$
}

\author{
Simone V. Gill ${ }^{1,2 \#}$, Michael K. Walsh ${ }^{2}$ \\ ${ }^{1}$ Boston University, Department of Occupational Therapy, Boston, USA; ${ }^{\#}$ Corresponding Author: simvgill@bu.edu \\ ${ }^{2}$ Department of Occupational Therapy, College of Health and Rehabilitation Sciences, Sargent College, Boston, USA
}

Received 10 November 2012; revised 15 December 2012; accepted 23 December 2012

\begin{abstract}
Obesity is an epidemic in the United States, and weight loss is key component to overcoming the problem. However, the risk of falling and injury in the obese population may hinder their engagement in the amount and intensity of physical activity needed to lose weight. The risks of falling and injury have been attributed to differences in temporospatial walking parameters, musculoskeletal structure, and cognitive processing: all of which affect the obese population's ability to adapt while walking. We have proposed that an intervention using motor learning principles with an implicit learning task could improve the adaptive abilities of obese individuals during movement and thus, allow for safer participation in physical activity during weight loss interventions.
\end{abstract}

Keywords: Obesity; Walking; Adaptation; Motor Learning

\section{INTRODUCTION}

The increased prevalence of obesity in adults from the 1970s through the 1990s [1] has made obesity a prime concern for health professionals in the United States for many years. Over the past decade, the rate of increase appears to be slowing or plateauing. However, numerous adults still have become and remain obese [2]; data from the National Health and Nutrition Examination Survey show that as of 2009-2010 approximately 36\% of US men and women are obese [3]. To combat the obesity epidemic, there has been an emphasis on encouraging physical and habitual (i.e. healthy eating, taking the stairs, etc.) lifestyle changes to aid in the weight loss process [4, 5]. While successful, these interventions take years to reach the desired goal because weight loss is a slow process $[4,5]$. Therefore, there is a need to design inter-

"Disclosures: Supported by Boston University Research Funds. ventions to support adults while they are losing weight.

Evidence shows that obesity is associated with differences in walking that affect the ability to adapt: the ability to modify movements when faced with changes in local conditions [6]. These differences may contribute to the greater incidence of falls and injuries seen in the obese population $[7,8]$. Differences in walking and subsequent risk of injury in the obese population suggest that they need more than just weight loss interventions. During the weight loss process, safety is also a priority. Therefore, weight loss interventions should take into consideration the increased risk of injury in individuals who are obese by examining and intervening on the impact of obesity on adaptation.

The purpose of this paper is to argue the importance of how an individual's ability to adapt affects safe participation in physical activity weight loss interventions. It is crucial that an aspect of obesity intervention include training adaptive abilities to reduce injuries and to allow for safer participation in physical activity interventions. Health professionals have the ability to address impairments in adaptation, however obesity interventions involving adaptive training have yet to be implemented. The ability to adapt can be key for obese adults to maintain safety during physical weight loss interventions.

\subsection{Review of Factors Affected by Adult Obesity}

Adult obesity is associated with impairments in many aspects of walking that affect adaptation, which can lead to falls and injuries [9]. For example, compared to normal weight adults, obese adults vary the timing and distance of their steps (i.e. temporospatial gait parameters) and produce smaller forces on the knee (reduced torque) during walking $[10,11]$. There are also positive correlations between adult obesity and knee osteoarthritis, and negative correlations between obesity and weakening bone strength $[12,13]$. Lastly, the obese population has been shown to perform poorly on cognitive tasks, including motor planning, which is important in adapting 
motor actions [14]. The links between obesity and impairments in movement, musculoskeletal structure, and cognitive processing all affect obese individuals' ability to adapt during walking and will be discussed further in this paper. Recognizing and addressing these areas when planning obesity interventions would allow participants to safely take part in physical activity and may increase their willingness to participate in physical activity due to increase self-confidence and self-efficacy [15].

\subsubsection{Impairments in Temporospatial Walking Parameters}

Many of the walking impairments related to adult obesity involve differences in temporospatial gait parameters between obese and normal weight individuals. To our knowledge, all of the research to date agrees there are walking impairments associated with adult obesity. When walking at a self-selected pace, compared to normal weight adults, adults who are obese have shorter stride lengths, walk more slowly (i.e. slower velocity), and spend more time with their feet in contact with the ground via longer double support times and stance times [6]. Obese adults also demonstrate decreased cadence (steps per minute), less time with their feet off the ground (i.e. shorter swing times), and walk with their feet farther apart with larger step widths $[11,16]$. Adult obesity is also associated with limited postural control and stability that could impair the ability to readily adapt while walking [17]. For example, obese men and women display a forward anterior posterior (AP) center of pressure position because they carry their weight towards the front of their feet, and increased AP instability during dynamic balance [17]. Therefore, the changes in temporospatial gait patterns have been interpreted as a way to compensate for instability during dynamic balance [11]. However, these changes, while intending to improve dynamic balance, may actually jeopardize the ability to recover when a loss of balance occurs and perpetuate patterns of walking that precipitate falling such as tripping [18] leading to more instances of falls.

Difficulty maintaining dynamic balance may be one reason for differences in walking parameters, but there is evidence that preserving skeletal health, particularly at the knee joint, may be another. These altered gait parameters lead to decreased knee torque in obese individuals [10]. The altered walking pattern acts as a mechanism for maintaining skeletal health with additional body weight due to obesity [10]. It is possible that although decreased torque via alterations in temporospatial gait parameters may help maintain skeletal health for obese individuals, it actually compromises their ability to maintain balance and recover from loss of balance. Regardless of the reason for the differences in walking, these impairments threaten obese adults' ability to adapt and capacity to prevent falls and injuries. They should be carefully considered during the creation of obesity intervention programs.

\subsubsection{Differences in Musculoskeletal Structure}

Aside from impairing walking, adult obesity also has a detrimental effect on musculoskeletal structure, which can hinder adaptation. Over the long term (e.g. up to 10 years), individuals with obesity are more likely to develop knee osteoarthritis than those who are not obese [19]. There is a dose-response relationship between the two variables, meaning that the greater the body mass index (BMI), the greater the likelihood of developing osteoarthritis [19]. The presence of osteoarthritis results in outcomes that affect walking and the ability to be adaptive. Increased knee pain has been associated with higher BMI scores: the higher the BMI the higher the degree of pain reported in the joint [12]. Therefore, the obese population is more likely to have osteoarthritis and to experience more joint pain than the normal weight population. While it has not been directly examined in the obese population, the increase in pain from knee osteoarthritis could result in a greater incidence of falls. For example, when artificial knee pain is induced in healthy individuals via a saline injection into the knee joint, adults demonstrate impairments in postural control and stability during quiet standing [20]. During induced unilateral knee pain, increased AP sway displacement was observed, and during bilateral knee pain, increased AP and medial-lateral (ML) sway displacement was noted [20]. Based on this finding, it is plausible that knee pain experienced by obese individuals could damage postural control and stability putting them at risk for loss of balance and falling.

Compromised bone strength is another musculoskeletal deficiency that has been observed in obese individuals. A number of studies pinpoint low bone mineral density in children who are obese, but no such studies have observed this in adults [21]. However, one study with eighteen and nineteen year old females showed a relationship between the percentage of body fat and the likelihood of experiencing deficits in overall bone strength. Bone failure can be predicted using the peripheral quantitative computed tomography (pQCT) method, which takes into account bone size, shape, and mineral density [13]. When adjusting for muscle cross-sectional area, the percentage of body fat was inversely related to pQCT measurements. Specifically, the high-fat group had significantly lower tibial and radial strength-strain indices, lower tibial cortical bone area and bone mineral content, and lower radial total bone cross-sectional area [13]. Contrary to prior research, this suggests that excess body weight is not advantageous to the skeletal apparatus, when the added weight is due to fat mass. While this 
research in young adults is in its preliminary stages, it lends credence to the idea that obese adults incur more injuries from falls because they 1) are more likely to fall; and 2) have weaker bones. These findings on osteoarthritis and bone strength highlight the importance for healthcare professionals to incorporate adaptation training into obesity interventions to address the safety of their participants.

\subsubsection{Limitations in Cognition}

Adult obesity may also affect adaptation by limiting an individual's ability to motor plan. Poor motor planning, the ability to pre-plan a movement before it is executed, leads to poor performance on tasks [22]. This could be a detriment to adaptation because adaptive behavior involves tailoring actions in the face of variability. Therefore, motor plans need to be changed during motor actions [23]. In the obese population, poor motor planning and an inability to change motor plans during the course of action, could lead to more frequent losses of balance or the inability to recover from unavoidable losses of balance.

While research on the relationship between obesity and cognition (e.g. motor planning) is relatively new, there is evidence that obesity negatively impacts performance on cognitive tasks. Compared to normal weight individuals, obese individuals (specifically with BMIs > 35) perform significantly worse on tests of executive function that involve planning and mental flexibility, both which are keys to motor planning [14]. Reasons for this relationship are not well understood at this point. However, possible causes include impaired metabolic processing affecting brain structures involved in planning and organization, such as the cerebellum [22]. Recent imaging studies suggest that obesity may be related to reduced cerebellar function in children and perhaps the same may hold true for adults [24]. Second, a lack of physical activity in the obese population could lead to a decrease in oxygen flow to the brain, which may impair spatial abilities needed to plan motor actions [25]. Each of these suggestions could negatively affect an obese individual's cognitive abilities and in turn, their motor planning. The inability to adapt quickly to change could lead to injuries and poses a serious safety risk for obese individuals.

\section{DISCUSSION}

Walking, musculoskeletal structure, and cognition all play a role in the ability to adapt. Training for the obese population focused on improving these areas involved in adaptation may help in their ability to modify motor movements needed to sustain the amount and intensity of physical activity to participate in obesity interventions.
Below we discuss how motor learning principles could be used to address the previously discussed deficits faced by the obese population in order to improve adaptation.

\section{Adaptation Training Intervention for Obese Adults}

An intervention using motor learning principles could help improve obese individuals' ability to adapt. Integrating the concept of implicit learning into these principles may be an effective way to improve adaptation. Implicit learning is defined as learning without the awareness of what is being learned [26]. In contrast, explicit learning is defined having a complete awareness of what is being learned [26]. Implicit learning has been shown to improve performance on cognitive motor tasks [27, 28]. Walking impairments caused by differences in temporospatial parameters (i.e. balance) and limits in cognition (i.e. poor motor planning) could benefit from training using an implicit task. The idea behind incorporating implicit learning into adaptation interventions is that, once learned, the task would become second nature so that there would be no need to think about how or when to perform it. The hope is that this would lead to improved adaptive abilities in obese adults and fewer incidences of falls and injuries. Implicitly learned plans have been shown to override explicitly learned strategies during visuomotor adaptive tasks [29]. Therefore, if a skill is learned implicitly, there is no need to rely on explicit strategies. Use of an implicit strategy would allow for readily adapting to changes during movement. Therefore, it makes sense to train the components of walking impaired by obesity, balance and cognitive function, with a implicit learning strategy.

Incorporating an implicit learning task with accepted motor learning principles, such as practice conditions, types of augmented feedback, and schedules of feedback, could be an effective way of constructing an adaptation intervention. These principles would help health professionals to structure practice and feedback for their clients to promote learning (See Table 1 for definitions and applications of principles). First, two types of practice conditions include blocked and random practice [30]. Blocked practice usually allows for better performance of a skill during practice because it is learned faster, but random practice has been shown to produce better retention of a skill after time away from practice even though performance during practice may suffer [30]. Second, augmented feedback falls into two categories, knowledge of results (KR) and knowledge of performance (KP). With $\mathrm{KR}$, the learner receives information about the outcome of performing a skill or task, whereas with $\mathrm{KP}$, information about the nature of the movement that led to the performance outcome is received [31]. Finally, sched- 
Table 1. Applied definitions of motor learning principles.

\begin{tabular}{|c|c|c|c|}
\hline Motor Learn & Principle & Definition & Example \\
\hline Conditions of Practice & Blocked Practice & $\begin{array}{l}\text { Repeating the same blocks of trials } \\
\text { throughout the practice session (i.e. A, A, } \\
\text { A, A, A; A = Easy, B = Medium, C = Hard ) }\end{array}$ & $\begin{array}{l}\text { Practicing all songs at the same difficulty } \\
\text { level throughout the entire practice session, } \\
\text { so the speed at which the participant must } \\
\text { process the visuomotor information remains } \\
\text { constant }\end{array}$ \\
\hline \multirow[b]{2}{*}{ Types of Feedback } & $\begin{array}{l}\text { Knowledge of } \\
\text { Results (KR) }\end{array}$ & $\begin{array}{l}\text { Feedback that gives information about the } \\
\text { task outcome }\end{array}$ & $\begin{array}{l}\text { Feedback that tells the participant whether or } \\
\text { not they stepped to the target in the desired } \\
\text { direction and timing }\end{array}$ \\
\hline & $\begin{array}{l}\text { Knowledge of } \\
\text { Performance (KP) }\end{array}$ & $\begin{array}{l}\text { Feedback that gives information about } \\
\text { the movement characteristics of the task } \\
\text { outcome }\end{array}$ & $\begin{array}{l}\text { Feedback given informing participants of } \\
\text { their body movements during losses of } \\
\text { balance or missteps and desired target hits. }\end{array}$ \\
\hline \multirow[t]{2}{*}{ Schedules of Feedback } & Intermittent & $\begin{array}{l}\text { Feedback given after the task is completed } \\
\text { (i.e. after every trial or after a few trials } \\
\text { in a row) }\end{array}$ & $\begin{array}{l}\text { Informing the participant of the number of } \\
\text { successful target hits or of their movement } \\
\text { characteristics after every song or every } \\
\text { few songs }\end{array}$ \\
\hline & Concurrent & $\begin{array}{l}\text { Feedback given during completion of the } \\
\text { task }\end{array}$ & $\begin{array}{l}\text { Informing participants of successful target } \\
\text { hits or of movement characteristics while } \\
\text { they are playing the game }\end{array}$ \\
\hline
\end{tabular}

ules of feedback can be intermittent or concurrent. This means that feedback can be given after the task is completed or feedback can be given during the task itself [32]. The goal of the intervention and the skill level of the participant should be considered when deciding which principles should be used. For example, it may be best to start beginners with blocked practice to familiarize them with the task and to promote adherence to the intervention. Once they are familiar with the skill, they can be transitioned to random practice, so that the skill can be retained and generalized to other aspects of movement.

A concrete example of how these strategies can be used to promote improvements in balance and cognitive function is with an interactive dance video game. It has been suggested that a game such as Dance Dance Revolution (DDR) (Konami) could be used in a clinical capacity to improve motor skills and cognition [33]. The game requires the player to stand on a sensor mat with pads that correspond to different step directions. The game scrolls directional arrows down a television screen indicating which pad the player should step on to the beat of a song. This task requires balance, timing, motor planning, and visuomotor integration: all of which are important to adapting while walking.

An intervention using DDR could include both blocked and random practice. Since blocked practice leads to less retention of skill, but faster skill acquisition, blocked practice can be used during the beginning stage of the intervention. Then, random practice can be introduced after a few sessions, to allow individuals to generalize the benefits of improved performance to everyday life. In this example, blocked practice could entail playing multiple songs in a row all at a preferred difficulty level, while random practice could involve playing songs above the preferred skill level every so often (i.e. interspersed randomly throughout the practice session). KR and KP elicit the same results with regard to motor learning, but the goal of an intervention focused on improving adaptation would be to encourage implicit learning [31]. Therefore, the focus of feedback in our proposed intervention would be based on movement outcomes rather than on the characteristics of movement related to performance. Using the DRR example, KR could be given about whether the player hit the correct direction on the mat with the correct timing while playing the game. Due to the nature of the game, feedback would be displayed concurrently. While literature on the use of intermittent vs concurrent feedback varies, there is evidence that concurrent feedback during rehabilitation is effective [34]. Therefore, the proposed intervention would include blocked and random practice, with continuous concurrent KR feedback.

To promote implicit learning, participants should focus on improving their scores (i.e. number of correct steps hit or points at the end of a session) rather than on how tocontrol balance or on memorizing the pattern of arrows presented during the game. The hope is that by taking 
part in an adaptation intervention that balance and cognition would improve without explicit effort and that implicit learning would strengthen an obese individual's ability to adapt.

\section{CONCLUSION}

Adult obesity is an epidemic. Healthcare professionals can play a major role in optimizing interventions to improve adaptation and to decrease the risk of falls and injuries in obese adults. Ultimately, weight loss is important, but it takes time. Interventions aimed at improving adaptation can be used in concert with overall obesity interventions focused on weight loss. Incorporating adaptation interventions into current obesity interventions can help ensure safe participation.

\section{ACKNOWLEDGMENTS}

The authors are grateful to the members of the Motor Development Laboratory for their helpful comments on an earlier draft of this manuscript.

\section{REFERENCES}

[1] Flegal, K.M., Carroll, M.D., Ogden, C.L. and Johnson, C.L. (2002) Prevalence and trends in obesity among US adults, 1999-2000. JAMA, 288, 1723-1727. doi:10.1001/jama.288.14.1723

[2] Flegal, K.M., Carroll, M.D., Ogden, C.L. and Curtin, L.R. (2010) Prevalence and trends in obesity among US adults, 1999-2008. JAMA, 303, 235-241.

doi:10.1001/jama.2009.2014

[3] Ogden, C.L., Carroll, M.D., Kit, B.K. and Flegal, K.M. (2012) Prevalence of obesity and trends in body mass index among US children and adolescents, 1999-2010. JAMA, 307, 483-490. doi:10.1001/jama.2012.40

[4] Goodpaster, B.H., Delany, J.P., Otto, A.D., Kuller, L., Vockley, J., South-Paul, J.E., Thomas, S.B., Brown, J., McTigue, K., Hames, K.C., Lang, W. and Jakicic, J.M. (2010) Effects of diet and physical activity interventions on weight loss and cardiometabolic risk factors in severely obese adults: A randomized trial. JAMA, 304, 1795-1802. doi:10.1001/jama.2010.1505

[5] Norris, S.L., Zhang, X., Avenell, A., Gregg, E., Schmid, C.H. and Lau, J. (2005) Long-term non-pharmacological weight loss interventions for adults with prediabetes. Cochrane Database of Systematic Reviews, 2, 1-14.

[6] Lai, P.P., Leung, A.K., Li, A.N. and Zhang, M. (2008). Three-dimensional gait analysis of obese adults. Clinical Biomechanics, 23, S2-S6. doi:10.1016/j.clinbiomech.2008.02.004

[7] Finkelstein, E.A., Chen, H., Prabhu, M., Trogdon, J.G. and orso, P.S. (2007) The relationship between obesity and injuries among US adults. American Journal of Health Promotion, 21, 460-468. doi:10.4278/0890-1171-21.5.460
[8] Hu, H.Y., Chou, Y.J., Chou, P., Chen, L.K. and Huang, N. (2009) Association between obesity and injury among Taiwanese adults. International Journal of Obesity, 33, 878-884. doi:10.1038/ijo.2009.122

[9] Wu, X., Lockhart, T.E. and Yeoh, H.T. (2012) Effects of obesity on slip-induced fall risks among young male adults. Journal of Biomechanics, 45, 1042-1047. doi:10.1016/j.jbiomech.2011.12.021

[10] DeVita, P. and Hortobágyi, T. (2003) Obesity is not associated with increased knee joint torque and power during level walking. Journal of Biomechanics, 36, 1355-1362. doi:10.1016/S0021-9290(03)00119-2

[11] Wearing, S.C., Hennig, E.M., Byrne, N.M., Steele, J.R. and Hills, A.P. (2006) The biomechanics of restricted movement in adult obesity. Obesity Reviews, 7, 13-24. doi:10.1111/j.1467-789X.2006.00215.X

[12] Marks, R. (2007) Obesity profiles with knee osteoarthritis: Correlation with pain, disability. Disease Progression, 15, 1867-1874.

[13] Pollock, N.K., Laing, E.M., Baile, C.A., Hamrick, M.W., Hall, D.B. and Lewis, R.D. (2007) Is adiposity advantageous for bone strength? A peripheral quantitative computed tomography study in late adolescent females. American Journal of Clinical Nutrition, 86, 1530-1538.

[14] Boeka, A.G. and Lokken, K.L. (2008) Neuropsychological performance of a clinical sample of extremely obese individuals. Archives of Clinical Neuropsychology, 23, 467-474. doi:10.1016/j.acn.2008.03.003

[15] McAuley, E. and Blisser, B. (2000) Self-efficacy determinants and consequences of physical activity. Exercise and Sports Sciences Reviews, 28, 85-88.

[16] Ko, S., Stenholm, S. and Ferrucci, L. (2010). Characteristic gait patterns in older adults with obesity-Results from the Baltimore Longitudinal Study of Aging. Journal of Biomechanics, 43, 1104-1110. doi:10.1016/j.jbiomech.2009.12.004

[17] Capodaglio, P., Cimolin, V., Tacchini, E., Parisio, C. and Galli, M. (2012) Balance control and balance recovery in obesity. Current Obesity Reports, 1, 166-173. doi:10.1007/s13679-012-0018-7

[18] Gill, S.V. and Narain, A, (2012) Quantifying the effects of body mass index on safety: Reliability of a video coding procedure and utility of a rhythmic walking task. Archives of Physical Medicine and Rehabilitation, 93, 728-730. doi:10.1016/j.apmr.2011.09.012

[19] Grotle, M., Hagen, K.B., Natvig, B., Dahl, F.A. and Kvien, T.K. (2008) Obesity and osteoarthritis in knee, hip and/or hand: An epidemiological study in the general population with 10 years follow-up. BMC Musculoskeletal Disorders, 9, 132. doi:10.1186/1471-2474-9-132

[20] Hirata, R.P., Arendt-Nielsen, L., Shiozawa, S. and GravenNielsen, T. (2012) Experimental knee pain impairs postural stability during quiet stance but not after perturbations. European Journal of Applied Physiology, 112, 25112521. doi:10.1007/s00421-011-2226-3

[21] Goulding, A., Taylor, R.W., Jones, I.E., McAuley, K.A., Manning, P.J. and Williams, S.M. (2000) Overweight and obese children have low bone mass and area for their 
weight. International Journal of Obesity, 24, 627-632. doi:10.1038/sj.ijo.0801207

[22] Wolpert, D.M. and Miall, R.C. (1996) Forward models for physiological motor control. Neural Netw, 9, 12651279. doi:10.1016/S0893-6080(96)00035-4

[23] Adolph, K.E., Joh, A.S., Franchak, J.M., Ishak, S. and Gill, S.V. (2008) Flexibility in the development of action. In: Morsella, E., Bargh, J.A. and Gollwitzer, P.M., Eds., The Psychology of Action, Oxford University Press, New York, 399-426.

[24] Miller, J.L., Couch, J., Schwenk, K., Long, M., Towler, S., Theriaque, D.W., He. G., Liu, Y., Driscoll, D.J. and Leonard, C.M. (2009) Early childhood obesity is associated with compromised cerebellar development. Developmental Neuropsychology, 34, 272-283. doi:10.1080/87565640802530961

[25] Dustman, R.E., Emmerson, R.Y. and Shearer, D.E. (1990) Aerobic fitness may contribute to CNS health: Electrophysiological, visual and neurocognitive evidence. Journal of Neurorehabilitation and Neural Repair, 4, 241254. doi:10.1177/136140969000400410

[26] Japikse, K.C., Negash, S., Howard Jr., J.H. and Howard, D.V. (2003) Intermanual transfer of procedural learning after extended practice of probabilistic sequences. $E x-$ perimental Brain Research, 148, 38-49. doi:10.1007/s00221-002-1264-9

[27] Howard, D.V. and Howard, J.H. (2001) When it does hurt to try: Adult age differences in the effects of instructions on implicit pattern learning. Psychonomic Bulletin \& Re- view, 8, 798-805. doi:10.3758/BF03196220

[28] Masters, R.S., Poolton, J.M., Maxwell, J.P. and Raab, M. (2008) Implicit motor learning and complex decision making in time-constrained environments. Journal of Motor Behavior, 40, 71-79. doi:10.3200/JMBR.40.1.71-80

[29] Mazzoni, P. and Krakauer, J.W. (2006) An implicit plan overrides an explicit strategy during visuomotor adaptation. Journal of Neuroscience, 26, 3642-3645. doi:10.1523/JNEUROSCI.5317-05.2006

[30] Simon, D.A. and Bjork, R.A. (2001) Metacognition in motor learning. Journal of Experimental Psychology Learning Memory and Cognition, 27, 907-912. doi:10.1037/0278-7393.27.4.907

[31] Winstein, C.J. (1991) Knowledge of results and motor learning-implication for physical therapy. Physical Therapy, 71, 140-149.

[32] Magill, R.A. (2007) Motor learning and control concepts and applications. McGraw-Hill, New York.

[33] Jovancevic, J., Rosano, C., Perera, S., Erickson, K.I. and Studenski, S. (2012) A protocol for a randomized clinical trial of interactive video dance: Potential for effects on cognitive function. BMC Geriatrics, 12, 23. doi:10.1186/1471-2318-12-23

[34] Intiso, D., Santilli, V., Grasso, M.G., Rossi, R. and Caruso, I. (1994) Rehabilitation of walking with electromyographic biofeedback in foot-drop after stroke. Stroke, 25, 1189-1192. doi:10.1161/01.STR.25.6.1189 